\title{
Model lung breathes true
}

An interesting calculation of how the human lung functions has appeared in an Italian journal whose fortunes appear (from inspection) to be reviving.

ONCE upon a time, in the $1950 \mathrm{~s}$, Il Nuovo Cimento shared with the Japanese journal Progress in Theoretical Physics the distinction of being among the most eagerly read in its field, but it was then eclipsed by the emergence of rapid-publication journals such as Physical Review Letters and, later, of Physics Letters.

But Il Nuovo Cimento seems to be perking up. Nowadays, it is almost always possible to find something of interest in an issue. Part of the explanation is that, for the past eleven years, there have been four sections dealing separately with nuclear physics, astrophysics, condensed matter and general physics, which means that there are in principle four issues a month. In some sections, the publication lag (one of the reasons for the original decline) is still uncomfortably long some papers seem to take a year in the process of publication But the more obscure backwaters of general relativity on which the journal retained a lien throughout its bleak period are no longer the staple diet for Il Nuovo Cimento's faithful readers. Last month's condensed matter issue, for example, contains an intriguing calculation of gas transport in the human lung of a kind that would normally have been found in a physiological journal.

As F. Bonsignori and M. Salvini, both physicists from the University of Bologna, describe it, the problem is really fairly straightforward (Il Nov. Cim. 11D, 1247; 1989). The trick, which has been used before, is to represent the lung and its airways, the trachea and bronchi, by a kind of one-dimensional model. They follow physiologists in supposing that there are 23 generations of successive refinement in the lung, at each of which the total area of the walls across which gas can be exchanged increases. The problem then becomes that of calculating the processes by which exchangable gases reach the walls of the lung and diffuse through them.

The physiological data used to ground the model are striking in themselves. The cross-section of the airways increases from $2.54 \mathrm{~cm}^{2}$ in the trachea to $11,800 \mathrm{~cm}^{2}$ at the finest and twenty-third subdivision of the alveoli, whose total volume is a third of the capacity of the lung as a whole (taken to be just over 1.5 litres, everything included). The most obvious consequence of this state of affairs is that gas velocities progressively decrease from the mouth to the alveoli, so that in the main airways into the lung, turbulent motion and convection are chiefly responsible for gas transport, but molecular diffusion dominates in the alveoli.

Bonsignori and Salvini acknowledge that their model is not original, but they do claim to have improved on earlier calculations (in the 1970s) by improving on some of the approximations on which previous calculations have been subject. For one thing, they reckon to have used a better representation of the cross section of the lung with distance along the airways. More pointedly, they allow for the way in which the effective diffusion constant can vary with the velocity of the flow and with place along the airways. In the alveoli proper, the implicit assumption is that the gases in the lung exchange their contents freely with the blood, which is for practical purposes regarded as an infinite reservoir.

The objective is to write down and then to solve a differential equation for the diffusion and convection of gases along the airways; Bonsignori and Salvini judiciously discuss the boundary conditions that should apply at the walls of the airways, acknowledging that in the alveoli, at least, there are grounds for believing that the concentration of one component of gas inspired will be virtually constant along directions perpendicular to the walls of the alveoli, and that from some point sufficiently deeply buried in the network, the concentration of all components will be virtually unchanging in space and time. But this implies that there are two formally different sets of boundary conditions that may be relevant, whence the suggestion (not further followed up) that some mixture of the two would be most appropriate.

The variations of the effective diffusion coefficient, which determines the effectiveness of mixing under different circumstances, are tackled semi-empirically. In the entering airways, mixing is much more efficient than would be determined by molecular diffusion, but in the alveoli, molecular diffusion is king. Part of the construction of the equations to be solved entails fixing parameters by reference to the known parameters of the lung, using the helpful (and reasonable) conditions that air should not accumulate anywhere in the system. The remarkable feature of the calculation is that it leads to an explicit result, although one in which several numerical approximations have been made.

The outcome of the calculation is much what physiologists would expect. The authors restrict themselves to the case in which it is supposed that somebody takes in a single substantial breath, holds it for a little while and then empties the lungs. What the solution of the equation shows is, first, that a component of the inspired gas progressively penetrates through the system with the passage of time, and that the process is the more rapid as the incoming flow is faster.

The supposition that, in the outer reaches of the system, the concentration of particular components of the inspired gas quickly become more or less constant readily falls out from the calculations, but concentrations in the inner reaches quickly fall to zero as gas is rapidly absorbed across the alveolar walls. The time constant of the system appears to be a few seconds - rather more than 4 seconds in one significant context - which accords with general experience of trying to hold one's breath. It may or may not be coincidence, but the calculations appear to show that, when breathing air, the model lung reaches in just over a second a condition in which the oxygen concentration is virtually constant throughout the inner part of the respiratory system, taken to be the last seven generations of airway refinement.

Inevitably, the authors promise to produce a second set of more refined calculations, paying particular attention to the vexed question of the boundary conditions most appropriate in different parts of the respiratory system. At this stage, however, nothing is said about the assumptions that the gas in the lungs is incompressible, or that there is no interference between the different components of the inspired gas that people breathe. No doubt the next step will be to collect some carefully controlled experimental data in the hope of matching calculation and experiment.

The interest of all this is not simply that it is what seems to be a reasonably realistic calculation of the properties of a complicated physiological system, but that it appears in a straightforward physics journal. No doubt the bibliographic retrievers will alert physiologists to what they may otherwise overlook, but that it should have appeared there at all is a neat sign of how broadly catholic is the Italian Physical Society.

John Maddox 